\title{
Structural transformation of graphite by arc-discharge
}

Article

Accepted Version

Authors final version published without significant changes after peer review

Harris, P. J. F. (2011) Structural transformation of graphite by arc-discharge. Philosophical Magazine, 91 (18). pp. 23552363. ISSN 1478-6443 doi:

https://doi.org/10.1080/14786435.2011.562250 Available at https://centaur.reading.ac.uk/24740/

It is advisable to refer to the publisher's version if you intend to cite from the work. See Guidance on citing.

To link to this article DOI: http://dx.doi.org/10.1080/14786435.2011.562250

Publisher: Taylor \& Francis

All outputs in CentAUR are protected by Intellectual Property Rights law, including copyright law. Copyright and IPR is retained by the creators or other copyright holders. Terms and conditions for use of this material are defined in the End User Agreement.

\section{www.reading.ac.uk/centaur}

\section{CentAUR}

Central Archive at the University of Reading

Reading's research outputs online 


\section{Structural transformation of graphite by arc-discharge}

\section{Peter J.F. Harris}

Centre for Advanced Microscopy, J.J. Thomson Physical Laboratory, University of Reading, Whiteknights, Reading RG6 6AF, UK.

e-mail: p.j.f.harris@rdg.ac.uk 


\section{Structural transformation of graphite by arc-discharge}

The formation of novel structures by the passage of an electric current through graphite is described. These structures apparently consist of hollow threedimensional graphitic shells bounded by curved and faceted planes, typically made up of two graphene layers. The curved structures were frequently decorated with nano-scale carbon particles, or short nanotubes. In some cases, nanotubes were found to be seamlessly connected to the thin shells, indicating that the formation of the shells and the nanotubes is intimately connected. Small nanotubes or nanoparticles were also sometimes found encapsulated inside the hollow structures, while fullerene-like particles were often seen attached to the outside surfaces. With their high surface areas and structural perfection, the new carbon structures may have applications as anodes of lithium ion batteries or as components of composite materials.

\section{Introduction}

Interesting things can happen when an electric current is passed through graphite. In 1990, Krätschmer and Huffman and their co-workers showed that $\mathrm{C}_{60}$ is formed, in high yield, by vaporising graphite in a simple carbon arc apparatus under an atmosphere of helium [1]. Most of the $\mathrm{C}_{60}$ is deposited on the walls of the arcevaporation vessel, along with disordered soot-like material. A short time later, Iijima showed that multiwalled carbon nanotubes with extremely perfect structures can be produced in a similar way [2], although in this case the nanotubes form on the negative graphite electrode. In 1993 it was shown that single-walled nanotubes can be produced by arc-discharge if $\mathrm{Co}$ and $\mathrm{Ni}$ or some other metal is added to the anode $[3,4]$.

Recently, yet another kind of carbon has been discovered in graphite samples through which a current has been passed [5]. This new carbon apparently consists of hollow graphitic shells bounded by curved and faceted planes, typically made up of two graphene layers. These structures were first found in a commercial ultra-pure graphite. It is believed that they formed during a purification process which involved passing an electric current through the graphite. Support for this idea was provided by 
some preliminary experiments involving the passage of a current through graphite rods in a carbon arc apparatus, which produced very similar structures. In the present study the controlled production of the novel graphene structures by the passage of an electric current through graphite is described in detail. The material is characterised using high resolution transmission electron microscopy, and evidence is presented that the structures are three-dimensional rather than flat. The possible mechanism whereby graphite could be transformed into the three-dimensional shells is discussed.

It is suggested that the new carbon structures might find applications in a wide variety of areas. For example, their extremely large surface-to-volume ratio and likely high conductivity suggest that they may be useful in sensors or lithium ion batteries. They may also have potential in catalysis or as hydrogen storage materials.

\section{Experimental}

The apparatus used to produce the new carbon structures was an Edwards S150B evaporation unit. This is normally used for coating scanning electron microscopy specimens with a thin layer of carbon. In this unit, the electrodes are 3 $\mathrm{mm}$ graphite rods, one of which is sharpened to a point and held in contact with the other electrode with a spring mechanism. High purity rods were used, with impurity content $<20$ ppm (Agar Scientific Ltd., UK). The chamber was pumped by a rotary pump, giving a vacuum of approximately 0.1 Torr. Discharge was carried out at a voltage of $10 \mathrm{~V}$, with a current of approximately $20 \mathrm{~A}$, for a duration of about 2 minutes. These are the standard conditions for carbon coating, although with a rather longer duration. Following arcing, the pointed electrode was generally found to have been shortened by a few mm, and a small deposit could be observed near the point of contact of the two electrodes. This deposit was scraped from the electrodes and then prepared for TEM by dispersing in iso-propanol and then pipetting onto holey carbon 
TEM films. A sample of a fresh graphite rod was also examined by TEM. The microscope used was a JEOL 2010, with a point resolution of $0.19 \mathrm{~nm}$, operated at an accelerating voltage of $200 \mathrm{kV}$. Images were recorded photographically.

\section{Results}

A micrograph of material from the fresh graphite rod is shown in Fig. 1(a). As expected, this consists mainly of flat crystallites, ranging from a few $100 \mathrm{~nm}$ to about $5 \mu \mathrm{m}$ in size, containing up to 100 layers. The crystallites were often folded and buckled, and were accompanied by small amounts of disordered material. However, nanotubes or other fullerene-related structures were not seen in the fresh graphite.

The carbon collected from the graphite rods following arcing contained some "normal" graphite, but this was accompanied by many regions which had a very different appearance. One of these areas is shown in Fig. 1(b). Here, the outline of the structure is much more irregular than in the fresh graphite, with many curved and unusually-shaped features. The material is decorated with numerous short nanotubes or nanoparticles. An area in which a number of nanotubes can be seen is shown in Fig. 2(a). These tubes typically possessed between two and four walls and could be several $100 \mathrm{~nm}$ in length, but tended to be less perfect than those grown by the traditional Iijima arc-evaporation method, in that they often contained sharp bends and other discontinuities. In some cases nanotubes were found to be directly connected to the larger structures; examples can be seen in this image. The observation that nanotubes are joined seamlessly to the larger regions indicates that these regions are three-dimensional rather than flat, the three-dimensional structure arising from presence of pentagonal and other non-hexagonal rings. The junctions between the tubes and the larger shell-like regions may involve seven-membered rings. 
A higher magnification image of another region is shown in Fig. 2(b). It can be seen from this image that the graphene material is largely bilayer, but some threelayer and single-layer graphene is also present. The single-layer graphene structures were found to be very beam-sensitive, so obtaining high quality images of this material proved difficult. The bilayer spacing, determined from this and other images, was generally somewhat larger than the interplanar spacing for graphite, being typically around $0.4 \mathrm{~nm}$.

The thin graphitic material exhibited many unusual features. In some areas, nanoparticles or nanotubes appeared to be encapsulated inside the large structures. This is illustrated in Fig. 3, where a single-walled nanoparticle can be seen apparently inside a bilayer shell. This provides further evidence that the thin graphitic structures are hollow. Another interesting observation is that very small particles, which appeared to be fullerenes, were frequently seen on the outside surfaces of the thin graphitic structures, as shown in Fig. 4. It is notable that the fullerene particle, which is approximately the size of a $\mathrm{C}_{60}$ molecule, is preferentially attached to the apex of the faceted graphene structure. The interaction of fullerenes with the conical graphene structures known as carbon nanohorns has been studied recently by Suarez-Martinez and colleagues [6]. These workers have shown that fullerenes are frequently observed at the tips of nanohorns, rather than on the main bodies of the structures. Although this phenomenon is not fully understood [7], it suggests that fullerenes prefer to become attached to regions containing pentagonal rings. Therefore, observations such as that in Fig. 4 suggest that the apex of the faceted structure contains a pentagon. This is supported by the fact that the apex makes an angle of approximately $150^{\circ}$. This is close to the angle observed in certain MWNT caps, where a conical region 
joins the cylindrical part of the tube (see ref. [8], p128). It is believed that a single pentagon occurs at the point where the two regions are joined.

\section{Discussion}

A remarkable transformation in the structure of graphite as a result of the passage of an electric current in an arc-discharge apparatus has been described. It is believed that this involves the formation of three-dimensional shell-like structures bounded by very thin walls. In most cases these walls consist of bilayer graphene, although walls with three or more layers are quite frequently seen. Single-layer graphene structures are also occasionally observed. There are a number of reasons for believing that these new structures are three-dimensional and hollow rather than flat. Firstly, as reported here and in the previous paper [5], carbon nanotubes are often observed to be seamlessly joined to the larger regions. It is difficult to envisage a way in which nanotubes, with their circular cross-section, could be connected to flat, few layer, graphene without being seriously distorted, at least in the vicinity of the junction. Secondly, nanoparticles or nanotubes are sometimes found encapsulated inside the large structures, as shown in Fig. 3, indicating that the structures are hollow. Thirdly, small fullerene particles, similar in size to $\mathrm{C}_{60}$ molecules, are often found preferentially attached to the apices of faceted graphene structures, as in Fig. 4, providing evidence for the presence of pentagonal rings.

Some structures rather similar to those described here have been reported in recent studies by Jia and co-workers [9] and by Huang et al. [10]. These studies involved in situ Joule heating of graphite "nanoribbons" inside a TEM. In both papers the authors drew a different conclusion about the nature of the transformation to that 
given here: the process was discussed in terms of sublimation and edge reconstruction of flat graphene. It is possible, however, that they were seeing a transformation from flat graphite to hollow structures, as described in the present work, rather than sublimation.

The mechanism of the transformation is not known at present, but a few comments can be made. It is possible that the key to understanding the process may lie in the edge structure of graphite planes. It is well established that graphite planes often have "closed" edges, so that the layers resemble folded sheets [11 - 15], as illustrated in Fig. 5 (a). In a sense, therefore, these adjacent graphene layers already represent closed structures, and the transformations reported in this paper may simply involve an "opening" of the layers, as shown schematically in Fig. 5 (b). Such a process might be initiated by the nucleation of pentagonal rings at the closed edges: it is clear from the presence of nanotube-like structures in the carbon, and the attachment of fullerenes to the faceted structures, that pentagons are present. It also seems clear that the electric current passing through the carbon, rather than simply the high temperature, is responsible for the transformation. However, further work is needed to gain a more detailed understanding of the process.

Although two-dimensional graphene has attracted huge interest in recent years $[16,17]$, the possibility of producing large three-dimensional structures with graphene walls has not been widely discussed. A few theorists have considered regular threedimensional networks built from small graphene patches (e.g. refs. [18 - 20]), but these require three sheets to be joined along a line, usually with $\mathrm{sp}^{3}$-bonded carbons, rather than two sheets as in the structures reported here. In 1995 Ebbesen and Hiura 
published a paper entitled "Graphene in 3-dimensions - towards graphite origami", but this was primarily concerned with using scanning probe microscopy to create folds in graphene layers on the surface of highly ordered pyrolytic graphite [21]. The resulting structures were still essentially two-dimensional. It has been suggested that chemical techniques could be used to create three-dimensional structures from graphene [22], or that nanoscale water droplets might induce graphene sheets to fold up like the petals of a flower [23, 24]. However, none of these ideas have yet been demonstrated experimentally. Currently, therefore, the technique described in this paper is believed to be the only method for synthesising large, hollow structures with ultrathin graphitic walls.

The new the three-dimensional graphene structures described here could potentially have important applications. One possible area might be hydrogen storage, since forms of carbon rather similar to those described here have been shown to have $\mathrm{H}_{2}$ storage capabilities. Thus, Orimo et al. reported in 1999 that "nanostructured graphite" prepared by mechanical milling of synthetic graphite can have high $\mathrm{H}_{2}$ uptakes [25]. Carbon nanohorns have also been shown to have potentially useful $\mathrm{H}_{2}$ storage properties [26]. Theoreticians have suggested that "pillared graphene", a three-dimensional network constructed from graphene and short nanotubes, could adsorb $6 \%$ of its weight in hydrogen at room temperature and pressure [27]. Although the present material is a disordered rather than an ordered network, its $\mathrm{H}_{2}$ storage characteristics might be similar to those of "pillared graphene".

The new structures may also have applications in electrical devices. Recently, it has been demonstrated that graphene can be used in electrochemical double layer 
capacitors [28]. The high surface area graphitic shells reported here might actually have advantages over conventional "flat" graphene in this application. The flat graphene particles would have a tendency to clump together, while the faceted shells would be more likely to retain their surface area. The high conductivity of the structures, and their large internal volume might also make them potential candidates for the anodes of lithium ion batteries [29].

Finally the mechanical properties of the graphitic shells might be superior in some ways to those of flat graphene. Although graphene sheets are extremely stiff in tension, they have little resistance to bending or compression. Curved or faceted structures would be expected to have much greater flexural rigidity. The new materials might therefore be useful components of composite materials.

\section{Acknowledgement}

I thank Kazu Suenaga for discussions.

\section{References}

[1] W. Krätschmer, L.D. Lamb, K. Fostiropoulos and D.R. Huffman, Nature 347 (1990) p.354.

[2] S. Iijima, Nature 354 (1991) p.56.

[3] S. Iijima and T. Ichihashi, Nature 363 (1993) p.603.

[4] D.S. Bethune, C.H. Kiang, M.S. de Vries, G. Gorman, R. Savoy, J. Vazquez and R. Beyers, Nature 363 (1993) p.605.

[5] P.J.F. Harris, J. Phys.: Condens. Matter 21 (2009) 355009.

[6] I. Suarez-Martinez, C.P. Ewels, J. Mittal and M. Monthioux, submitted for publication (2011).

[7] I. Suarez-Martinez, M. Monthioux and C.P. Ewels, Journal of Nanoscience and Nanotechnology 9 (2009) p.6144. 
[8] P.J.F. Harris, Carbon Nanotube Science, Cambridge University Press, Cambridge, 2009.

[9] X. Jia, M. Hofmann, V. Meunier, B.G. Sumpter, J. Campos-Delgado, J.M. Romo-Herrera, H. Son, Y.P. Hsieh, A. Reina, J. Kong, M. Terrones and M.S. Dresselhaus, Science 323 (2009) p.1701.

[10] J.Y. Huang, F. Ding, B.I. Yakobson, P. Lu, L. Qi and J. Li, Proceedings of the National Academy of Sciences of the United States of America 106 (2009) p.10103.

[11] D. Ugarte, Chem. Phys. Lett. 198 (1992) p.596.

[12] P.R. Buseck, H. Bo-Jun and L.P. Keller, Energy and Fuels 1 (1987) p.105.

[13] H. Murayama and T. Maeda, Nature 345 (1990) p.791.

[14] Y. Gogotsi, J.A. Libera, N. Kalashnikov and M. Yoshimura, Science 290 (2000) p.317.

[15] Z. Liu, K. Suenaga, P.J.F. Harris and S. Iijima, Phys. Rev. Lett. 102 (2009) 015501 .

[16] A.K. Geim and K.S. Novoselov, Nature Materials 6 (2007) p.183.

[17] A.K. Geim, Science 324 (2009) p.1530.

[18] N. Park and J. Ihm, Phys. Rev. B 62 (2000) p.7614.

[19] K. Umemoto, S. Saito, S. Berber and D. Tománek, Phys. Rev. B 64 (2001) 193409.

[20] T. Kawai, S. Okada, Y. Miyamoto and A. Oshiyama, Phys. Rev. B 72 (2005) 035428.

[21] T.W. Ebbesen and H. Hiura, Advanced Materials 7 (1995) p.582.

[22] P.M. Ajayan and B.I. Yakobson, Nature 441 (2006) p.818.

[23] N. Patra, B. Wang and P. Král, Nano Letters 9 (2009) p.3766.

[24] V.H. Crespi, Nature 462 (2009) p.858.

[25] S. Orimo, G. Majer, T. Fukunaga, A. Züttel, L. Schlapbach and H. Fujii, Appl. Phys. Lett. 75 (1999) p.3093.

[26] H. Tanaka, H. Kanoh, M. El-Merraoui, W.A. Steele, M. Yudasaka, S. Iijima and K. Kaneko, J. Phys. Chem. B 108 (2004) p.17457. 
$\begin{array}{ll}\text { Figure } 2 & \text { (a), (b) Typical structures found in carbon following arc-discharge. }\end{array}$

Figure 3 Bilayer graphene structure, apparently hollow with single-walled

Figure 5 Schematic illustration of transformation of folded graphene sheets into hollow structure. (b) Micrograph at same magnification showing transformation in structure following arc-discharge.

Figure $1 \quad$ (a) Low magnification micrograph of carbon from fresh graphite rod. nanoparticle inside (arrowed).
Figure $4 \quad$ Faceted bilayer graphene structure with enlarged region showing small fullerene particle attached to apex.

[27] G.K. Dimitrakakis, E. Tylianakis and G.E. Froudakis, Nano Letters 8 (2008) p.3166.

[28] M.D. Stoller, S.J. Park, Y.W. Zhu, J.H. An and R.S. Ruoff, Nano Letters 8 (2008) p.3498.

[29] G.X. Wang, X.P. Shen, J. Yao and J. Park, Carbon 47 (2009) p.2049.

\section{Figure Captions}



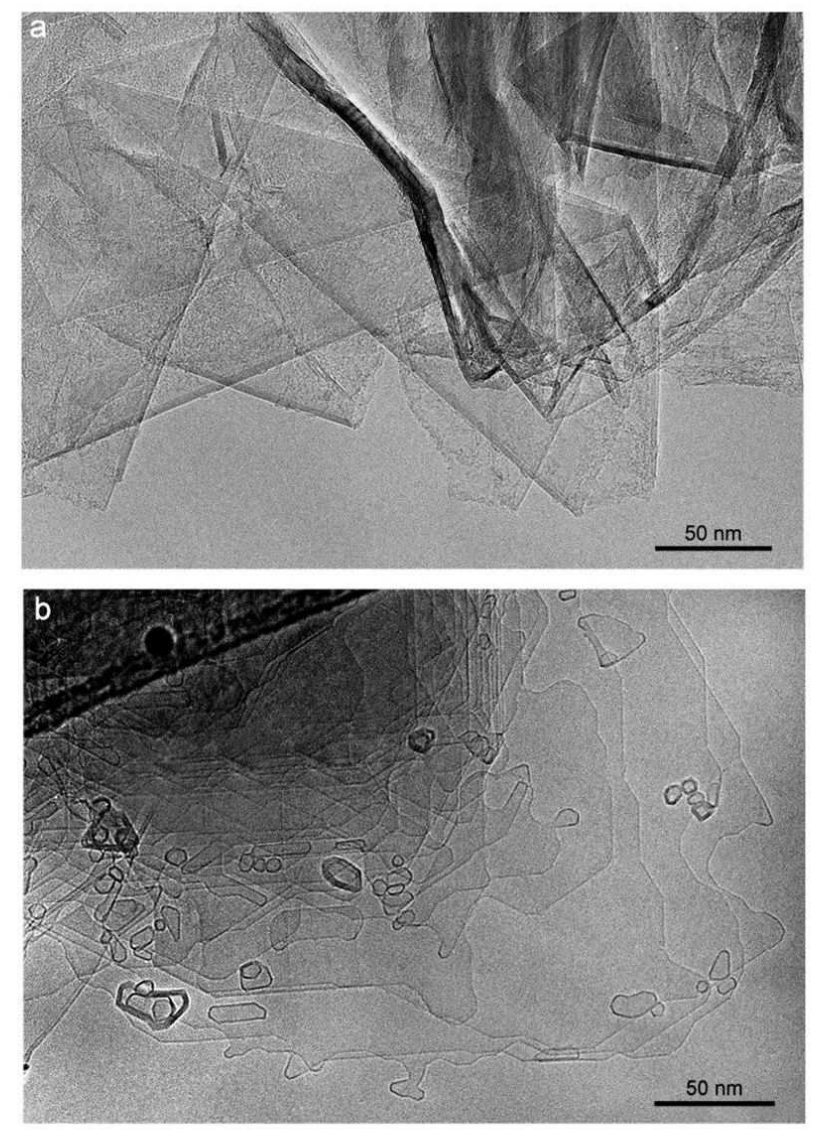

Fig. 1

(a) Low magnification micrograph of carbon from fresh graphite rod. (b) Micrograph at same magnification showing transformation in structure following arc-discharge. $179 \times 287 \mathrm{~mm}(139 \times 139 \mathrm{DPI})$ 

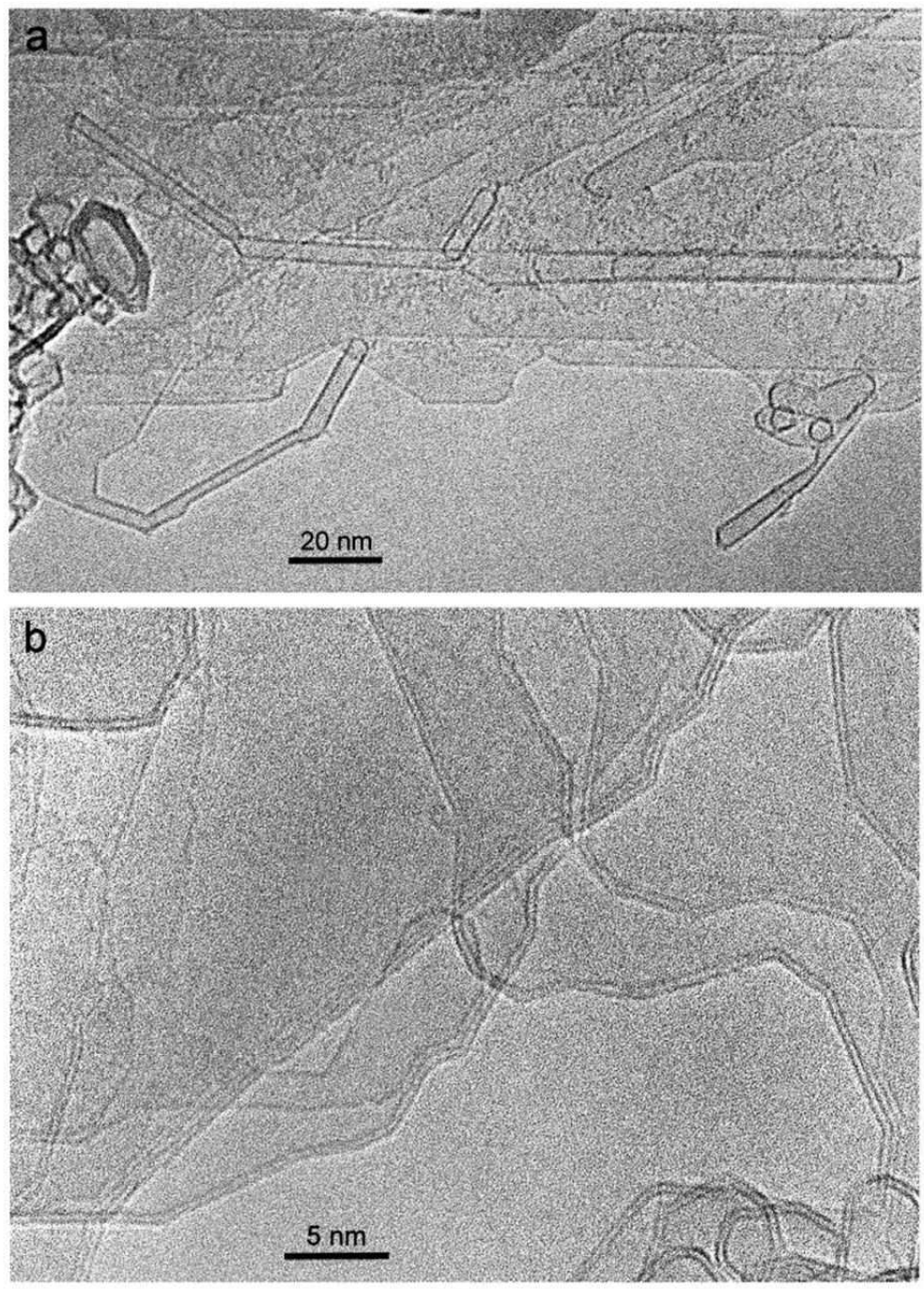

Fig. 2

Typical structures found in carbon following arc-discharge. $199 \times 266 \mathrm{~mm}(139 \times 139 \mathrm{DPI})$ 


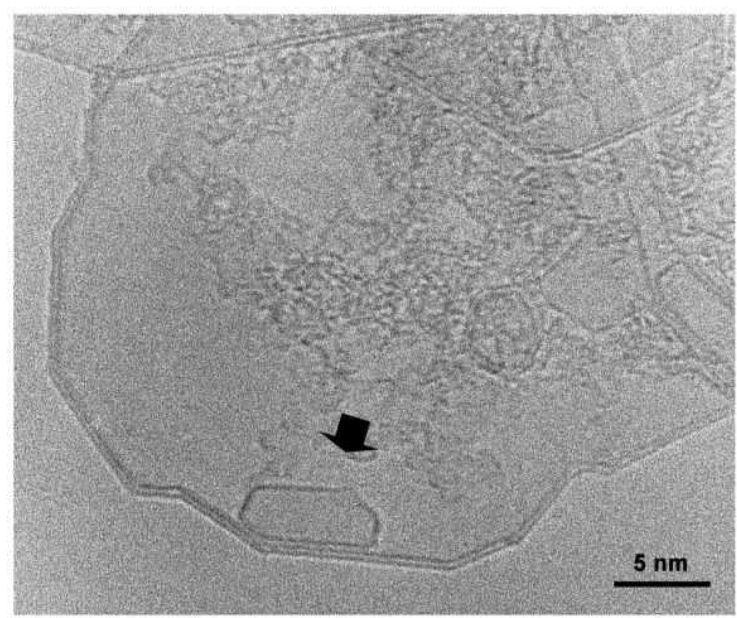

Fig. 3

Bilayer graphene structure, apparently hollow with single-walled nanoparticle inside (arrowed). $169 \times 226 \mathrm{~mm}(139 \times 139 \mathrm{DPI})$ 


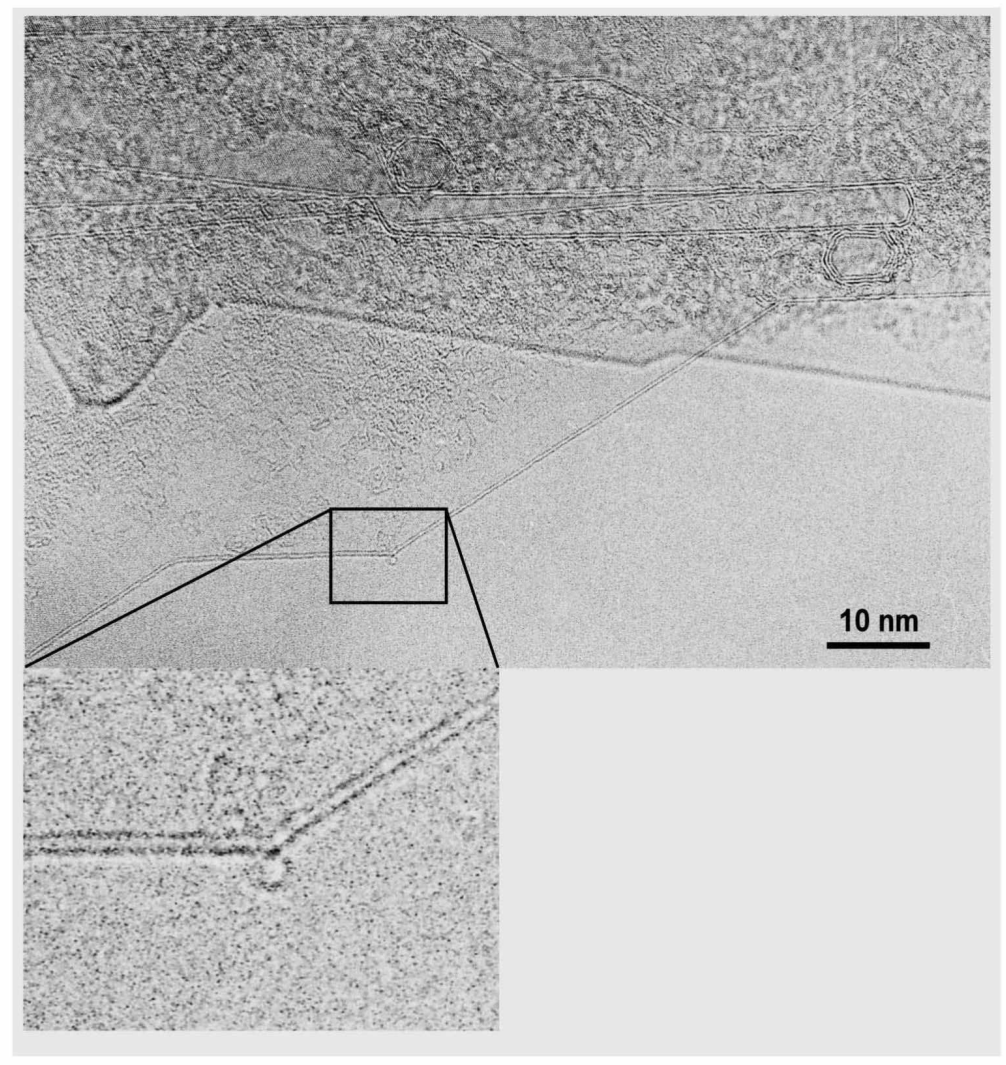

Fig. 4

Faceted bilayer graphene structure with enlarged region showing small fullerene particle attached to apex. $180 \times 180 \mathrm{~mm}(281 \times 281 \mathrm{DPI})$ 


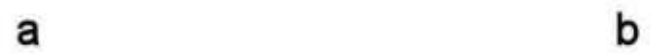

\section{Schematic illustration of transformation of folded graphene sheets into hollow structure. $110 \times 146 \mathrm{~mm}(139 \times 139$ DPI $)$}

Fig. 5

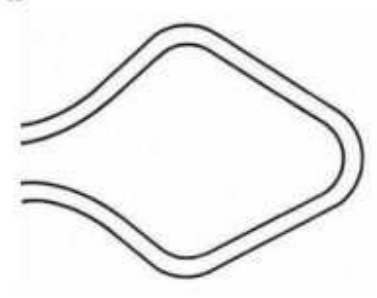

